\title{
LES GOITRES BÉNINS EN ORL ASPECTS EPIDEMIOLOGIQUES ET ANATOMOCLINIQUES : ÉTUDE DE 97 CAS
}

\author{
M. KEITA, D. DIANGO*, C. TRAORÉ**, B. KAMATÉE*, A. AG MOHAMED \\ SERVICE ORL, HÔPITAL GABRIEL TOURÉ \\ * SERVICE DE RÉANIMATION MÉDICALE, HÔPITAL GABRIEL TOURÉ \\ ** LABORATOIRE D'ANATOMIE PATHOLOGIQUE. INRSP \\ BAMAKO. MALI
}

\begin{abstract}
But : Analyse épidémiologique, clinique et anatomopathologique des goitres bénins.
Méthodologie : Etude rétrospective de janvier 2002 à décembre 2006 sur dossier de patients ayant consulté en ORL pour goitre. Les paramètres suivant ont été étudiés :

- interrogatoire : âge, sexe, profession, résidence, ancienneté du goitre, motif de consultation ;

- examen clinique précisant résultats palpation, classification OMS de 1962 du goitre ;

- résultats de l'échographie ;

- résultats du dosage de la TSH ultrasensible.

Ont été exclus tous les dossiers comportant une histologie suspecte et/ou maligne et les inflammations de la thyroïde. Ainsi a-t-on retenu 97 dossiers sur 122.

Résultats : Notre recrutement a été marqué par une forte proportion d'adulte jeune (classe d'âge de 31 à 50 ans : 78 patients) et par une prédominance féminine (sex-ratio, 17/80).

Ces patients étaient plus souvent des femmes au foyer et résidaient le plus souvent à Bamako.

Ils étaient porteurs à $52 \%$ de goitre évoluant depuis plus de vingt ans et à $44 \%$ de plus de dix ans d'évolution. Deux signes principaux ont amenés les patients à consulter : les signes d'hyperthyroïdie et la tuméfaction cervicale disgracieuse.

Le goitre était de type 3 de l'OMS dans $68 \%$ des cas. Un aspect multi nodulaire clinique a été vu chez 81 patients. A l'imagerie, nous avons noté $10 \%$ de goitre isoéchogène. L'examen anatomopathologie a plus souvent trouvé des goitres de type macro folliculaire sur les pièces d'exérèse. Ils étaient dans $20 \%$ des cas des goitres en activité.

Conclusion : Le praticien ORL en pays d'endémie goitreuse se doit de connaitre tous les contours de la pathologie thyroïdienne. II ne doit jamais oublier non plus que la prise en charge efficiente doit être multidisciplinaire (Endocrinologue, Radiologue, Anatomopathologiste, Anesthésiste Réanimateur).
\end{abstract}

Mots clés : Thyroïde, goitre bénin, tuméfaction cervicale.

Goal: epidemiological, clinical and anatomopathological analysis of benign goiter

Patients and methods : retrospective study of 97 cases traeted from January 2002 to December 2006. Were excluded malignant and inflammatory thyroid deseases.

Results: Our patients were essentially young adults (from 31 to 50 years: 78 patients). We noted a female prevalence (sex-ratio: 17/80)

$52 \%$ had more than twenty years evolution goiter and $44 \%$ more than ten years evolution. Two principal symptoms led the patients to consult: those of hyperthyroidy and cervical swelling. The goiter was classified as type 3 of WHO in $68 \%$ of the cases. A multi nodular aspect was found among 81 patients. We noted $10 \%$ of isoechogene goiter. The treatment was surgery The histological examination found macrofollicular goiter in the majority of cases.

Key words : Thyroïd gland, goiter bénign, cervical swelling.

\section{INTRODUCTION}

Le goitre est une hypertrophie diffuse de la thyroïde, due à une hyperplasie des cellules folliculaires. C'est une affection très répandue dans le monde. On distingue les 
goitres endémiques et les goitres sporadiques, selon des critères épidémiologiques. Un goitre est dit endémique lorsqu'il atteint plus de $10 \%$ de la population adulte dans une région donnée. Un goitre est dit sporadique dans le cas inverse. Cette définition regroupe les goitres avec ou sans signe de dysthyroïdie (7).

Le goitre endémique est la première endémie mondiale (7). La carence iodée a été reconnue comme étant la cause principale du goitre endémique. II concerne 1,5 milliards de personnes, reparties sur l'ensemble du globe terrestre dont une partie en Afrique.

Le Mali est dans la ceinture du goitre endémique et le goitre y constitue un véritable problème de santé publique. Ainsi a-t-on des zones au centre du pays ou la prévalence est de plus de $50 \%(2,12)$.

Cette étude a pour but de mener une analyse épidémiologique, clinique et histologique de cette pathologie thyroïdienne, tumeur bénigne la plus fréquente du pays (2).

\section{MATÉRIEL ET MÉTHODES}

A travers une étude rétrospective, de janvier 2002 à décembre 2006, ont été inclus tous les patients ayant consulté en ORL pour goitre et dont l'histologie définitive a confirmé l'absence de nature maligne et dont les dossiers d'observation contenaient les données suivantes :

- un interrogatoire ayant précisé : l'âge, le sexe, la profession, la résidence, l'ancienneté du goitre et les motifs de consultation ;

- un examen clinique précisant les résultats de la palpation, la classification OMS du goitre de 1962 (stade 0 : sujet non goitreux ; stade 1 : sujet goitreux, avec thyroïde palpable, mais non visible ; stade 2 : goitre visible mais dont le volume est inférieur à une balle de tennis ; stade 3 : goitre volumineux) ;

- les résultats de l'échographie ;

- les résultats du dosage de la TSH Ultrasensible.

Ont été exclus tous les patients présentant une histologie suspecte et/ou de type malin et les inflammations de la thyroïde.

Nous avons ainsi pu colligé 97 dossiers, et exclus 25 dossiers à histologie maligne.

\section{RESULTATS}

\section{Données sociodémographiques :}

Résidence : Soixante patients résidaient à Bamako, trente quatre résidaient dans cinq provinces du Mali (Kayes, Koulikoro, Sikasso, Mopti et Gao). Les villes du Nord du Mali ont été presque absente du recrutement à part Gao (1 cas).

Quatre patients étaient de Nationalité étrangère.

Sexe : Il s'agissait de quatre vingt femmes contre dix sept hommes. (Tableau I)

Profession : Cinquante patientes étaient femmes de ménage et/ou paysannes, huit sans profession, sept élèves et/ou étudiants, dix huit employés de bureau, dix cadre moyen, deux cadres supérieurs.

\begin{tabular}{|c|c|c|c|c|c|c|}
\hline $\mathrm{Sexe}^{\text {age }}$ & $\begin{array}{l}0-20 \\
\text { ans }\end{array}$ & $\begin{array}{l}21-30 \\
\text { ans }\end{array}$ & $\begin{array}{l}31-40 \\
\text { ans }\end{array}$ & $\begin{array}{l}41-50 \\
\text { ans }\end{array}$ & $\begin{array}{l}51 \text { ans } \\
\text { et plus }\end{array}$ & Total \\
\hline homme & 0 & 2 & 6 & 8 & 1 & 17 \\
\hline femme & 1 & 10 & 27 & 37 & 5 & 80 \\
\hline Total & 1 & 12 & 33 & 45 & 6 & 97 \\
\hline
\end{tabular}

Tableau I : Répartition des goitres par classe d'âge et par sexe

\section{Données cliniques :}

a. Motif de consultation :

Nos patients ont consulté essentiellement pour des motifs tels que :

- Tuméfaction cervicale disgracieuse : 77

- Signes d'hyperthyroïdie : 75

- Signes de compression (dysphagie, toux, dyspnée, Claude Bernard Horner, parésie ou paralysie recurrentielle): 4

b. Ancienneté du goitre : Elle était de plus de 20 ans dans 52 cas soit $53 \%$, entre 10 et 19 ans dans 44 cas (soit $45 \%$ ) et dans un cas de moins de 10 ans d'évolution (2\%).

\section{c. Résultats de la palpation :}

- Consistance : nous avons trouvé chez 71 patients une consistance bien ferme, chez 26 patients une consistance molle;

- Recherche de nodule : un aspect multi nodulaire a été retrouvé dans 81 cas et dans 16 cas un aspect sans nodule palpable; aucune adénopathie cliniquement décelable n'a été notée.

d. Classification OMS : soixante six de nos patients étaient porteurs de goitre de type 3 (68\%), vingt sept de type 2 (28\%) et quatre de type 1 (4\%).

e. Résultats de l'échographie :

- Isoéchogène : 10

- Hyperéchogène : 64 soit $66 \%$ des cas.

- Hétérogène : 13

- Non Fait : 10

f. Statut hormonal* : (Tableau II)

- Hyperthyroïdie : 79

- Euthyroïdie 10

- Hypothyroïdie : 8

\begin{tabular}{|c|c|c|c|c|c|c|}
\hline statut Age & $0-20$ & $21-30$ & $31-40$ & $41-50$ & $\begin{array}{l}51 \text { ans et } \\
\text { plus }\end{array}$ & Total \\
\hline Hyperthyroïdie & 0 & 9 & 27 & 40 & 3 & 79 \\
\hline Euthyroïdie & 1 & 2 & 2 & 2 & 3 & 10 \\
\hline Hypothyroïdie & 0 & 1 & 4 & 3 & 0 & 8 \\
\hline Total & 1 & 12 & 33 & 45 & 6 & 97 \\
\hline
\end{tabular}

Tableau II : Statut hormonal en fonction de l'âge

* Norme utilisée : TSH ultrasensible (2ème génération) :

Euthyroïdie : limite inférieure 0,20 à $0,50 \mathrm{mU} / \mathrm{l}$, limite supérieure 3,00 à $5,00 \mathrm{mU} / \mathrm{l}$

Hyperthyroïdie : < limite basse de la normale $:<0,10$ à $0,40 \mathrm{mU} / \mathrm{l}$

Hypothyroïdie : > limite haute de la normale : > 4,00 à 6,00 mU/I 


\section{Données histologiques :}

Les resultats de l'examen histologique des pièces d'exérèse chirurgicales sont résumés par les tableaux suivants (tableaux III et IV) :

\begin{tabular}{l|lc} 
Type histologique & Effectif & Fréquence en \% \\
\hline Macrofolliculaire & 75 & 77 \\
\hline Microfolliculaire & 6 & 6 \\
$\begin{array}{l}\text { Macro et } \\
\text { microfolliculaire }\end{array}$ & 16 & 17 \\
\hline Total & $\mathbf{9 7}$ & $\mathbf{1 0 0}$
\end{tabular}

Tableau III : Répartition en fonction du type histologique

\begin{tabular}{l|ll} 
Type de Remaniement & Effectif & Fréquence en \% \\
\hline Sans remaniement & $\mathbf{7 7}$ & $\mathbf{8 0}$ \\
\hline Hémorrag ique & 7 & 7 \\
\hline Fibro-hémorragique & $\mathbf{3}$ & $\mathbf{3}$ \\
\hline Fibreux & 7 & 7 \\
\hline Edème & $\mathbf{2}$ & $\mathbf{2}$ \\
\hline Calcification & $\mathbf{1}$ & $\mathbf{1}$ \\
\hline Total & $\mathbf{9 7}$ & $\mathbf{1 0 0}$ \\
\hline & Tableau IV : Répartition en fonction du remaniement \\
histologique
\end{tabular}

Répartition en fonction de l'activité :

La majeure partie de nos patients ( $80 \%$ soit 77 malades) ne présentaient pas un goitre en activité.

Répartition du goitre en fonction du statut hormonal et de l'état d'activité : (tableau V)

- Etat d'hyperthyroïdie : dans ce lot de 79 patients seuls 19 patients présentaient un goitre en activité ;

- Etat d'euthyroïdie : sur 10 patients, deux patients présentaient un goitre en activité ;

- Etat d'hypothyroïdie : 2 patients sur 7 présentaient un goitre en activité.

\begin{tabular}{|c|c|c|c|c|}
\hline \multirow[t]{2}{*}{ Type histologique } & \multicolumn{3}{|c|}{ Statut hormonal } & \multirow[t]{2}{*}{ Tota } \\
\hline & hyperthyroidie & euthyroidie & hypothyroidie & \\
\hline Macrofollicule & 72 & 3 & 8 & 83 \\
\hline Microfollicule & 2 & 0 & 0 & 2 \\
\hline $\begin{array}{l}\text { Macro et } \\
\text { microfollicule }\end{array}$ & 5 & 7 & 0 & 12 \\
\hline Total & 79 & 10 & 8 & 97 \\
\hline
\end{tabular}

Tableau V : Répartition du goitre en fonction du type histologique et du statut hormonal

\section{DISCUSSION}

\section{a. Aspects sociodémographiques} Age/Sexe:

Dans notre série, 90 patients sur 97 avaient un âge inférieur ou égal à 50 ans avec des extrêmes allant de 2 ans à 72 ans. Quand au sexe, le même constat a prévalu par rapport au sexe féminin qui est surreprésenté à hauteur de quatre vingt femmes contre dix sept hommes.

Ce constat concernant la prédominance du sexe féminin et d'une tranche d'adulte de moins de 50 ans confirme les constatations de plusieurs auteurs africains $(2,4,9,10,12,14)$ et d'ailleurs $(1,8)$.

Résidence : Notre recrutement a été marqué par une prédominance de patients résidant surtout à Bamako (60 patients sur 97) et dans quatre autres provinces du mali à savoir Koulikoro, Kayes, Sikasso, Mopti (36 patients).

Les trois régions nord du pays, situées en zone désertique n'ont presque pas été intéressées à part Gao d'où nous est venu une patiente.

Cette provenance semble obéir à la répartition de l'endémie goitreuse du Mali qui a jusqu'à présent épargné ces zones désertiques (12).Cependant n'oublions pas que le site de recrutement qu'a été notre service est aussi situé à Bamako.

\section{b. Aspects cliniques :}

Nos patients ont consulté pour les motifs principaux suivants souvent imbriqués :

- une demande de réparation de préjudice esthétique due à la tuméfaction cervicale disgracieuse ;

- des signes gênant d'hyperthyroïdie à type de tachycardie, palpitations, trouble du rythme, troubles du caractère. Ces signes ont très souvent donné un itinéraire thérapeutique particulier à ces patients avec des sollicitations diverses de praticiens ;

Assez rarement des signes de compression (4 cas) à type de dysphagie, toux, et un cas de syndrome de Claude Bernard Horner (enophtalmie, ptosis, myosis), ont motivé une consultation ORL sur avis d'autres confrères.

Nos patients ont été porteurs pour près de $98 \%$ de cas de goitre anciens, sans signes cliniques au début. A l'admission le goitre volumineux était prédominant ( $96 \%$ des cas, type 3 et 2 de l'OMS) et le plus souvent dans un contexte d'hyperthyroïdie ( 79 cas soit $81 \%$ des cas). Cette prédominance du contexte hyperthyroïdien pourrait être expliqué par la longue durée d'évolution de nos cas. Il est ainsi reconnu que l'hyperthyroïdie survient sur les goitres hétérogènes anciens comportant des zones devenues plus ou moins autonomes, plus ou moins indépendantes de la TSH $(1,3,5,6,11)$.

\section{c. Aspects échographiques}

L'exploration échographique de nos goitres orientait plus souvent vers la bénignité. En effet, selon la littérature (13) l'aspect hyperéchogène orienterait vers la bénignité, l'aspect hétérogène oriente vers un adénome ou un kyste tandis que l'aspect isoéchogène évoquerait quelques rares fois un goitre malin. 


\section{d. Aspects anatomopathologiques}

Nous avons constaté que le goitre macrofolliculaire a été prédominant dans notre série (83 cas /97). Ce constat corrobore déjà les conclusions d'une étude antérieure malienne (2).

II est aussi admis ( 7) que l'unité fondamentale de la thyroïde est le follicule, structure sphérique creuse formée d'un épithélium uni stratifié reposant sur une mince lame conjonctive et limitant une lumière contenant une substance visqueuse, la colloïde, secrétée par les cellules folliculaires. Ces cellules folliculaires montrent des variations de tailles et de forme en rapport avec l'activité fonctionnelle de la glande. Le diamètre moyen des follicules est de 200 micromètres. Les follicules au repos sont volumineux (200-500 micromètres), à épithélium aplati, avec une colloïde abondante. Au contraire, les follicules en état d'hyperactivité sont plus petits (30-50 micromètres) à épithélium cylindro-cubique, centrés par une lumière rétractée contenant une colloïde pâle.

Nous avons eu des cas de remaniements avec évolution vers des formes hémorragiques, fibreuses et même calcifié.
Selon la littérature les remaniements de type hémorragique ainsi que les calcifications, en particulier les cristaux d'oxalate (7) n'auraient pas de signification pathologique.

Notons cependant qu'un certain nombre (vingt malades soit $20 \%$ des cas) de nos patients présentaient un goitre en activité lors de l'examen de la pièce thyroïdienne.

\section{CONCLUSION}

Le goitre en pratique ORL est de plus en plus une réalité. Et, le praticien se doit d'en connaître tous les contours même si la prise en charge pluridisciplinaire (ORL, endocrinologue, radiologue et anatomopathologiste) est une nécessité de tous les jours.

\section{REFERENCES}

1. Ashizawa K

Epidemiology of Basedow disease and other thyroid diseases

Nippon Rinsho. 2006 ;64(12):2194-200

2. Bagayoko T

Les goitres bénins dans le service de chirurgie $B$ à l'hôpital National du Point G Thèse de Médecine Bamako, 1999

3. Christophe P, Christoph A.M.

Nodules Thyroïdiens et goitres: le traitement chirurgical

Schweiz Med Forum 2004 ; 4 :1090-1096.

4. Drabo Y.J., Dembele S.M., Ouandaogo J., Ouiminga R.M.

Problème du goitre endemique

Cas de 3 villages du Departiment de Tibga (Gourma-Burkina Faso)

Médecine Afrique Noire, 1992, 39(11):737-740.

5. Franklin JA

Lack of consensus in Europe in the management of nontoxic multinodular goitre. Clin Endocrinol (Oxf), 2000;53: 3-4

6. Freitas JE.

Therapeutic options in the management of toxic and non-toxic nodular goiter. Semin Nucl Med, 2000; 30: 88-97.

7. Leclère J., Orgiazzi J. Rousset B., Schlienger J.L., Wémeau J.L.

La thyroïde : des concepts à la pratique.

Paris, Edition Elsevier, 2ème edition, 2001,618p

8. Montury S., de Clermont H, Gatina J.H

Prévalence des cancers thyroïdiennes à la Réunion à partir des données scintigraphiques
Bull Patho Exot, 1997

9. Moumen M, Touhami M, Elaoui M.E, Fares F.El

Les micro-carcinomes thyroïdiens

Médecine du Maghreb, 1992, $31: 25-28$.

10. Ntyonga-Pono M.P

La pathologie thyroïdienne du Gabonais en milieu hospitalier à libreville : étude de 137 cas.

Bull Patho Exotique, 1998 ; 91(3) :pp

11. Pinchera A, Aghini-Lombardi F, Antonangeli L, Vitti P.

Multinodular goiter. Epidemiology and prevention

Ann Ital Chir. 1996;67(3):317-25.

12. Sidibe AT, Sidibe S, Ag Mohamed et coll

Interêt de la cytoponction au cours de nodules thyroïdiens en zone d'endémie goitreuse

Mali Médical 2000, $15: 25-29$

13. Tagni-Zukami D, Yomi J, Monkam G, Dzongang T, Bengono-Obe L, GonsuFotsin J.

Aspects Echographiques de la pathologie hypertrophique du corps thyroïde à l'Hôpital Militaire de Yaounde

Médecine d'afrique noire, 1996, 43(3) :136-141.

14. Toure A., Diallo A.T, Camara L.M, Toure F.B., Camara N.D.

La chirurgie Thyroïdienne : Expérience du service de Chirurgie Générale du

CHU Ignace DEEN de Conakry

Mali Médical, Tome 21(3) :23-27. 